\title{
Assessment of occupational risks of employees engaged in open-pit mining
}

\author{
S. S. Timofeeva ${ }^{1 *}, I . V$. Drozdova $^{1}$, and A. A. Boboev ${ }^{2}$ \\ ${ }^{1}$ Irkutsk National Research Technical University, 664074, Irkutsk, Russian Federation \\ ${ }^{2}$ Navoi State Mining Institute, 706800, Navoi, Uzbekistan
}

\begin{abstract}
Currently, Uzbekistan is in the process of legislation modernization, primarily in matters of labor protection and industrial safety. Modernization consists in the transition from a previously existing ineffective system focused on compensation payments to a modern system based on preventive approaches, which imply continuous improvement of work conditions. The aim of this work is to analyze the legislation in the field of labor protection management, obliging employers to proceed to occupational risks assessment and their management, as well as the assessment of occupational risks for miners, working under the influence of harmful and dangerous production factors during gold mining in the quarry, and the development of measures to reduce them.

The possible reasons for the discrepancy with the stated requirements of the physical factors of the miners working environment when performing work in gold mining quarries are analyzed. It has been established that professional risks for personnel are classified as high and medium and it is necessary to carry out organizational and technical measures to minimize them.
\end{abstract}

\section{Introduction}

Rapid changes taking place in the economy, production and business in the context of globalization have a significant impact on the technosphere safety, and above all, on labor protection sphere. Acceleration of scientific and technological progress, rapid accumulation of new scientific data leads to the development of integration processes and the need to apply unified approaches to assess working conditions at industrial enterprises. Modern companies develop and implement integrated management system that meet the requirements of international standards ISO 9001, OHSAS 18001 and ISO 14001, and further ensure its continuous operation. One of the requirements of such a system is to preserve the health and efficiency of personnel - the most valuable resource of the enterprise. The employer must take all possible measures to prevent and eliminate the causes that lead to accidents and occupational diseases. In order to prevent the onset of a dangerous situation, it is necessary to investigate potential causes that may lead to negative consequences. That is why the organization should have a professional risk management system that will allow you to identify and assess professional risks and develop measures to minimize them.

\footnotetext{
${ }^{*}$ Corresponding author: sstimofeeva@mail.ru
} 
The government of Uzbekistan pays considerable attention to the development of national legislation and its improvement on the basis of international instruments, such as international conventions. Today, Uzbekistan has already ratified 13 international conventions, including Convention No. 187 - on the framework for promoting occupational safety and health, Geneva 2006 and No. 188 - on the protection of employees from occupational risks caused by air pollution, noise and vibration at the workplace Geneva 1977, including Convention No. 161 - on occupational health services, Geneva - 1991.

The main law of Uzbekistan in the field of labor protection is No. 3PY-410 dated 22.09.2016. "On labor protection", where the directions of the state policy in the field of labor protection are fixed. In addition, Uzbekistan has adopted a number of by-laws regulating the procedure for certifying workplaces, methods for establishing classes of work conditions and guidelines for assessing occupational risks. The Ministry of Health of the Republic of Uzbekistan is responsible for developing a methodology for occupational risks assessment. International practice and practice in Russia and Uzbekistan show that the level of danger in the occupational environment is not only not decreasing, but it is growing year by year, and it is necessary to create a system for occupational risks management.

The term "occupational risk" as the probability of causing harm to health as a result of exposure to industrial factors in Uzbekistan was legislated in April 2017. In March 2007, Uzbekistan, among eleven CIS countries, adopted the interstate standard GOST 12.0.2302007, which fully complies with the principles of the International Labour Organization (MOT/ILO), set out in the Manual on occupational safety management systems (MOTСУОТ2001), as well as in the ILO Framework Convention No. 187. In the development of this standard, since July 1, 2018, Uzbekistan has been among the countries that voted for the adoption of interstate standards GOST 12.0.230.4-2018 and GOST 12.0.230.5 -2018 "System of labor safety standards. Occupational health and safety management systems", which describe in detail the procedures for identifying hazards and methods for occupational risks assessment.

According to international standards, the implementation of production processes and labor operations that are inextricably linked with dangerous and harmful production factors and other hazards, basically, can not be absolutely safe for the employee. Since there is almost always an accidental possibility of a situation when the impact of hazards on the body of the employee becomes unavoidable.

Such random possibilities of adverse events, taking into account the significance of their consequences, are called risks, and their identification, analysis and evaluation is mandatory and central issue within the framework of the occupational safety management system. The standards provide methodological tools for application of risk analysis methods.

Since it is not possible to create a single universal method suitable for organizations of different types of economic activity, the standard lists the general approaches and universal principles, algorithms, applications. Each organization has the right to develop and to apply risk assessment techniques taking into account the specifics of its activity.

The risks to which the personnel of enterprises are exposed according to GOST are divided into the following categories:

- exposure risks, which are a combination of the accidental possibility of exposure to a hazard on the employee's body and the significance (severity) of the consequences of such exposure;

- situational risks (risks of initiating): a combination of the accidental possibility of occurrence and the significance (severity) of the consequences of a dangerous situation in which the risk of exposure is not excluded, and in which new hazards may appear with corresponding risks of exposure or initiation.

The risk assessment procedure consists in determination of the risk degree and assigning it with a certain rank of the ordinal scale, point or verbal. 
Risk ranking is a procedure for ordering ranking objects in descending or ascending order of any of their qualitative properties when measured in the ordinal scale.

The degree of risk is a measure of risk, point and/or verbal, that ranks the place of this risk among other risks under ordinal scale. According to the degree of risk, there are:

- negligible risk level: the degree of risk that can be ignored and, without taking any special measures to ensure safety, allow personnel to perform work performed within the framework of general measures of safe behavior and safe working practices, almost without the use of specially provided measures and means of safety ensuring.

- acceptable risk level (acceptable risk): the degree of such risk, in which the organization can allow employees to perform work, but only with strict compliance with the established rules for performing work and the use of regulated security measures and tools;

- unacceptable risk level (unacceptable risk): the degree of such a high socially significant risk, in which the organization can not allow personnel to perform work under the applicable work regulations, regulated measures and security measures due to the possibility of a serious accident.

As a result of the risk assessment, the organization should obtain:

- maximum objective information about the state of working conditions, existing hazards and risks of their impact on employees;

- ordered lists of risks, ranked by risk level, allowing to identify the most vulnerable points of labor safety, to develop step-by-step measures for risk management and reliable labor safety of employees;

- the most detailed information for making informed decisions on risk management and allowing to develop and implement preventive and regulatory measures to protect employees from risks in order of priority.

The purpose of this work is to assess occupational risks for mining employees who are engaged in the conditions of exposure to harmful and dangerous production factors during gold mining in the quarry, and to develop measures to reduce them.

\section{Research methods}

As the source data, the materials of workplace certification were used performed for the divisions engaged in work in the quarry at the Murutau deposit. The work assessed the working conditions of personnel at each stage of the mining life cycle, namely: a bulldozer driver, a car driver (engaged in transporting rock mass in the technological process), an excavator driver, an explosives expert, a drilling rig driver, a pumping plant driver, and a crane driver.

Identification of hazards and assessment of working conditions - negative production factors at the workplace was carried out in accordance with the guidelines adopted in Uzbekistan [1]. Directly at the workplace, a set of production factors was assessed: dust - aerosols of mainly fibrinogenic action, toxic substances, noise, infrasound, common and local vibration, adverse parameters of the microclimate and light environment, psycho-physiological factors: the severity and intensity of work. Factors can vary in a wide range during the shift and often exceed the maximum permissible values. The general assessment of working conditions at the workplaces of these professions is characterized by a harmful third class with varying hazard degree. The leading factors are noise-vibration, dust on the background of an unfavorable microclimate.

According to statistics, in recent years, there has been an increase in the registration of occupational diseases, the main mass of which is vibration disease, which is determined by increased levels of vibration at the workplace, exceeding the maximum permissible levels by 3-10 dB; as well as an increase in the equivalent corrected level of vibration speed at the workplace of drilling rig, excavators drivers and drivers of heavy-duty dump trucks. The negative consequences are amplified by the 
microclimate of workplaces, work in forced and uncomfortable positions, and the entry of gases into the air of the working area: carbon monoxide, nitrogen oxides, sulfur dioxide, hydrogen sulfide and aerosols formed during drilling and blasting operations [2-5].

The risk assessment was based on GOST 12.0.230.4-2018 and 12.0.230.5 and risk analysis methods $[6,7]$.

\section{Results of the study and their discussion}

The technological process at ore gold mining pits is shown in Fig. 1.

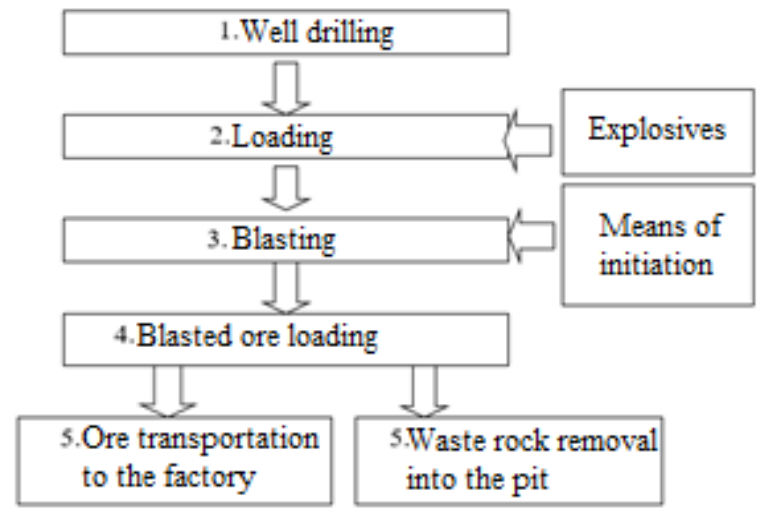

Fig. 1. Scheme of the technological process at the mining pits

The personnel who ensure the implementation of technological processes are affected by a complex of adverse factors of the working environment and work load of chemical, physical and psycho-physiological nature. The dust factor is represented by dust of complex chemical composition with the predominance of aerosols of mainly fibrinogenic action. As a rule, the $\mathrm{SiO}_{2}$ content ranges from 10 to $70 \%$. The air of the working area receives harmful gases from blasting operations, working transport, as well as the release of gases from rocks. The personnel involved in transporting the rock are exposed to a combination of physical factors such as noise, infrasound, common and local vibration, and there are significant sensory and emotional loads. Mining workers are also exposed to adverse microclimates combined with significant physical exertion.

One of the simplest methods recommended for occupational risks assessment is the matrix method. The essence of which is to determine the degree of risk on the matrix as a combination of the frequency or probability of occurrence of negative consequences, and the potential consequences themselves. In this case, the frequency of the event and the consequences themselves are determined by the expert method. However, this method is largely subjective, and the working conditions of mining employees are extremely dangerous and require objective information. This information is obtained during the performance of the workplace certification procedure, when the impact factors are directly measured by monitoring devices and compared with hygiene standards and the establishment of work conditions classes.

Currently, a forecast assessment of occupational risks based on the summary statement of work conditions has been performed. The method of forecast risk assessment involves an assessment of the generalized level of safety of the production environment, related to work experience. The maximum acceptable level of risk is calculated from the condition that all 
factors of the production environment that affect the employee in the course of work are brought to the best level. Ideally, these are work conditions classes for each factor that are optimal or acceptable, with the exception of those factors that cannot be reduced (improved) due to a particular technological process (for example, noise from equipment $[8,9]$.

Based on instrumental measurements of the actual parameters of the production environment and their comparison with hygienic standards, we determined the generalized level of safety of the production environment using the point method.

The generalized level of safety at each workplace is equal to the product of all levels of safety for each factor of the production environment for a particular profession.

The values of generalized occupational risk levels for each working profession for an open-pit mining site are given in Fig. 2.

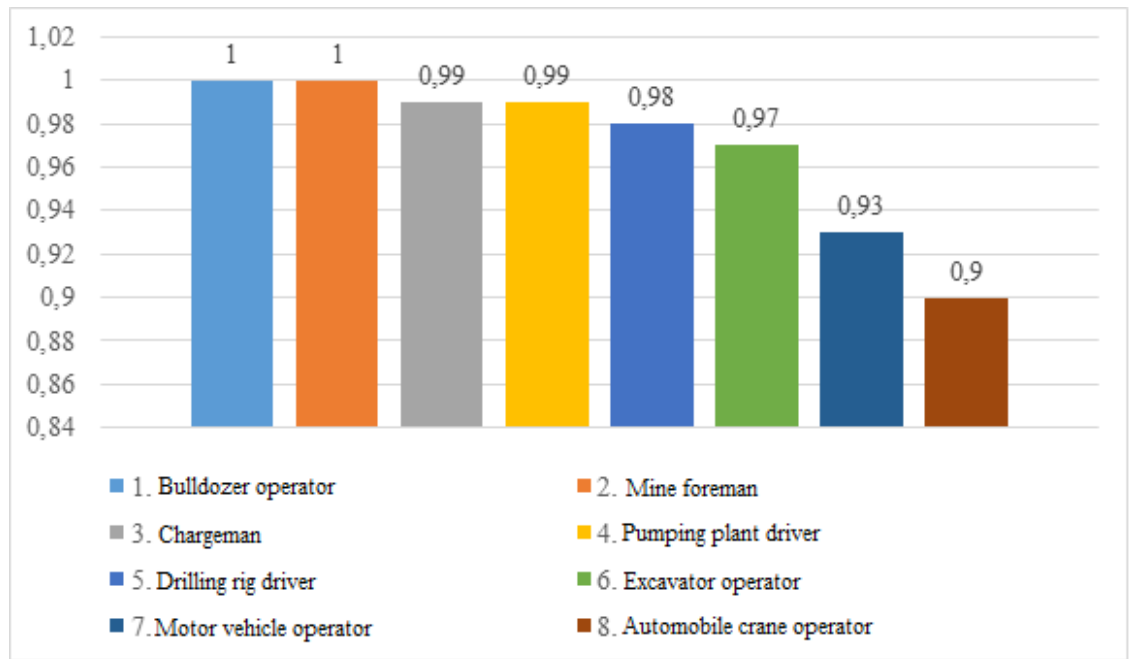

Fig. 2. Ranking of employee's professions for an open-pit mining site by the generalized level of professional risk

It is established that professional risks for personnel are classified as high and medium and it is necessary to carry out organizational and technical measures to minimize them.

Among the technical measures, first of all, it is mandatory to monitor the current operation of equipment and quarry equipment, including maintenance and repairs, to implement automated control systems for the mining and transport complex, such as "Wenco".

It is possible to ensure the safety of miners not only by using PPE (personal protective equipment), but also new digital technologies. The main direction of development of digital technologies for labor protection is training of personnel in an easy and accessible form to act in normal and emergency operation [10].

Today, it is impossible to imagine a good employee who does not know how to use a smartphone and does not have enough applications on it for storing and instant data processing. This applies to the sphere of labor protection [11]. There are a huge number of applications for smartphones related to ensuring compliance with the requirements of labor protection, industrial safety, civil defense and emergency situations, etc. It is only necessary to choose the program most suitable for the enterprise and follow its notifications.

Teaching-supervising system "OLYMPOCS" allows to automate training and testing of knowledge in the field of occupational health and safety, as well as a number of related processes: reporting, planning, notification of employees, and conducting briefings [11]. 
The system structure includes five main sections: "Exam preparation", "Exam", "Competition", "Introductory instruction", and "System management". It includes the following blocks:

- A system of personal accounts that displays a set of assigned training courses / areas of certification and exam deadlines

- Management of schedules of knowledge checks for each employee (dates of upcoming and repeated exams, appointment of an unplanned knowledge check, etc.)

- Sending notifications about the upcoming exam deadlines by email, including notifications of violation of the exam deadline

- Self-training in the mode of ready-made training courses studying, passing exams in the form of testing, forming protocols and reports on the results of exams

- Conducting introductory instructions in electronic form, photo-recording of employees, automatic maintenance of an electronic log of instructions

- Conducting competitions in the format of an electronic quiz on the knowledge of the requirements of regulatory documentation in a particular area

- Assignment of compulsory self-training and monitoring of the progress of studying individual topics and courses for each employee

- Self-study for exams using mobile devices and the OLIMPOCS mobile application (free on Google Play and the App Store) [11].

\section{References}

1. U. H. Adilov, Universum: Medicine and Pharmacology. Elecr. Sci. Journ, 1 (46), (2018) http://7 universum.cjm.ru/med/archive/inten/5446

2. I. V. Bukhtiyarov, N. P. Golovkova, A. G. Chebotarev, A. A. Salnikov, Occupational Medicine and Industrial Ecology, 5, 44-49 (2017)

3. Jacqueline Castelo Branco, Rebbah Rania, J. Duarte, João Santos Baptista, Risk Occupational and Environmental Safety and Health, 13-21 (2019) doi: 10.1007/978-3030-14730-3_2.

4. A. Badri, S. Nadeau, A. Gbodossou, J. of Safety and Security Eng, 2 (2), 145-156 (2012)

5. S. S. Timofeeva, M .A. Murzin, Safety in the technosphere, 3 (48), 37-42 (2014)

6. S. S. Timofeeva, E. A. Khamidullina, Fundamentals of risk theory: the workshop. city of Irkutsk: Publishing house of the Irkutsk State Technical University, 150 (2014)

7. S. S. Timofeeva, Methods and technologies of industrial risk assessment: Practical works for undergraduates, 177 (Irkutsk: Publishing house of the Irkutsk State Technical University,2013)

8. S. S. Timofeeva, XXI century. Technosphere security, 1 (1), 14-24 (2016)

9. S. S. Timofeeva, S. S. Timofeev, A. A. Boboeev, Uzbekistan Mining Bulletin, 2 (81), 107-111 (2020)

10. Business games on labor protection, Blog-Engineer URL:https://xn----8sbbilafpyxcf8a.xn--p1ai/\%D0\%B8\%D0\%B3\%D1\%80\%D1\%8B

11. Educational and controlling services OLYMPOCS URL: https://online.olimpoks.ru/katalog-kursov.php 